\title{
459 - Psychometric properties of the Geriatric Anxiety Inventory : A systematic review
}

Philippe Landreville; Alexandra Champagne; Patrick Gosselin

Background. The Geriatric Anxiety Inventory (GAI) is a widely used self-report measure of anxiety symptoms in older adults. Much research has been conducted on the psychometric properties of the GAI in various populations and using different language versions. Previous reviews of this literature have examined only a small proportion of studies in light of the body of research currently available and have not evaluated the methodological quality of this research. We conducted a systematic review of the psychometric properties of the GAl.

Method. Relevant studies $(\mathrm{N}=30)$ were retrieved through a search of electronic databases (Pubmed, PsycINFO, CINAHL, EMBASE and Google Scholar) and a hand search. The methodological quality of the included studies was assessed by two independent reviewers using the "COnsensusbased Standards for the selection of health status Measurement INstruments" (COSMIN) checklist.

Results. Based on the COSMIN checklist, internal consistency and test reliability were mostly rated as poorly assessed (62.1\% and $70 \%$ of studies, respectively) and quality of studies examining structural validity was mostly fair ( $60 \%$ of studies). The GAl showed adequate internal consistency and test-retest reliability. Convergent validity indices were highest with measures of generalized anxiety and lowest with instruments that include somatic symptoms. A substantial overlap with measures of depression was reported. While there was no consensus on the factorial structure of the GAl, several studies found it to be unidimensional.

Conclusions. The GAI presents satisfactory psychometric properties. However, future efforts should aim to achieve a higher degree of methodological quality. 